\title{
A Model of Emotion as Patterned Metacontrol
}

\section{Ricardo Sanz and Guadalupe Sánchez}

\author{
ASLab A-2011-020 v 0.0 Draft
}

December 21, 2011

\begin{abstract}
Adaptive systems use feedback as a key strategy to cope with uncertainty and change in their environments. The information fed back from the sensorimotor loop into the control architecture can be used to change different elements of the controller at four different levels: parameters of the control model, the control model itself, the functional organization of the agent and the functional components of the agent. The complexity of such a space of potential configurations is daunting. The only viable alternative for the agent ?in practical, economical, evolutionary terms? is the reduction of the dimensionality of the configuration space.

This reduction is achieved both by functionalisation - or, to be more precise, by interface minimization - and by patterning, i.e. the selection among a predefined set of organisational configurations. This last analysis let us state the central problem of how autonomy emerges from the integration of the cognitive, emotional and autonomic systems in strict functional terms: autonomy is achieved by the closure of functional dependency.

In this paper we will show a general model of how the emotional biological systems operate following this theoretical analysis and how this model is also of applicability to a wide spectrum of artificial systems.
\end{abstract}

Keywords: Cognitive architecture, Functional organisation, Emotion, Adaptive Systems, Autonomous Systems. 


\section{Contents}

1 Emotion research 3

1.1 A Modeling Flow . . . . . . . . . . . . . . . . . . . 3

1.2 Motivation for emotion in technical systems . . . . . . . . . . . . . 4

2 Emotion in vivo and in silico $\quad 5$

2.1 The many facets of emotion . . . . . . . . . . . . . 5

2.2 Four core aspects of emotion . . . . . . . . . . . . . 5

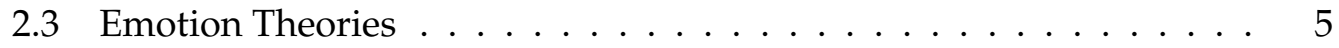

2.4 Emotion beyond biology . . . . . . . . . . . . . . 6

2.5 Emotion beyond biology . . . . . . . . . . . . . . . 6

2.6 A Stepped Program . . . . . . . . . . . . . . . . . 6

$\begin{array}{lll}3 & \text { Adaptive systems } & 7\end{array}$

3.1 Emotion-Cognition-Autonomy . . . . . . . . . . 7

3.2 The issue of adaptation . . . . . . . . . . . . 7

$\begin{array}{llr}4 & \text { Reconfiguration in Cognitive Systems } & 7\end{array}$

4.1 Variable Structure Controllers . . . . . . . . . . . . . . . . . . . 8

4.2 Change and Timescale $\ldots \ldots \ldots \ldots$

4.3 Structural Adaptation . . . . . . . . . . . . . . . . 10

4.4 Fight or Flight . . . . . . . . . . . . . . . . . . . 10

4.5 Architectural Change . . . . . . . . . . . . . . . . . . 10

4.6 Architectural Patterns . . . . . . . . . . . . . . . . 10

4.7 Taming the adaptation problem . . . . . . . . . . . . . . 11

4.8 Two basic processes . . . . . . . . . . . . . . . . . . . 11

4.9 Anatomy /Physiology . . . . . . . . . . . . . . . . 13

5 Emotion as pattern-based metacontrol $\quad 13$

5.1 What patterns to activate? . . . . . . . . . . . . 13

5.2 A View on Emotion . . . . . . . . . . . . . . . . . . . . . . 13

5.3 Emotion and Feedback . . . . . . . . . . . . . . . . . . . . . 14 
5.4 The Emotion Bus . . . . . . . . . . . . . . . . . . . . . . 14

5.5 Two Cognitive Loops . . . . . . . . . . . . . . . . . . . . . . 15

5.6 Heterophenomenology . . . . . . . . . . . . . . . . . . . . 15

$\begin{array}{llr}6 & \text { Science and technology } & 15\end{array}$

6.1 Model Evaluation . . . . . . . . . . . . . . . . . . . . 15

6.2 Mapping into Machines . . . . . . . . . . . . . . . . . 15

6.3 Cognitive Functional Metacontrol . . . . . . . . . . . . . . . 16

6.4 Technological path . . . . . . . . . . . . . 16

6.5 Current state . . . . . . . . . . . . . . . . . . 16

6.6 Problems and issues $\ldots \ldots \ldots \ldots \ldots$

6.7 Conclusions . . . . . . . . . . . . . . . . . . . . 17

\section{Emotion research}

\subsection{A Modeling Flow}

Computer implementations of cognitive models are common research assets in cognitive science (Sun, 2008). These models are created to better understand the modelled biological process by both a) the rigorous study that is necessary for building such a computer model and b) by the evaluation testbed for the model that a running program constitutes. Figure 1 shows an activity flow when developing cognitive models using computer programming. These models can be built from scratch as conventional programs or can often be implemented using programmable cognitive modeling engines like ACT-R or SOAR (Anderson et al., 2004). These systems are realisations of general cognitive architectures. These implementations of general theories of mind are used to create more specific models of concrete psychological phenomena.

The process, whether using conventional programming or the domain specific languages of these engines, starts with the selection of a concrete cognitive aspect to be evaluated - visual target tracking, spoken word recognition in cocktail parties, fear of bears, etc. - and continues with the proposal of a concrete model that can be translated into a computer implementation.

Obviously not all cognitive models described in the literature comply with this requirement. Many of them are expressed in too weak terms as to be easily translatable into programs. In other, even more extreme cases, the described models contain not just fuzzy but non-computable and/or metaphysical elements that are a-priori non-translatable to computer programs. Implementations are a filter of 
weak theories; a filter that is considered too strict by some, because it rules out non-computational

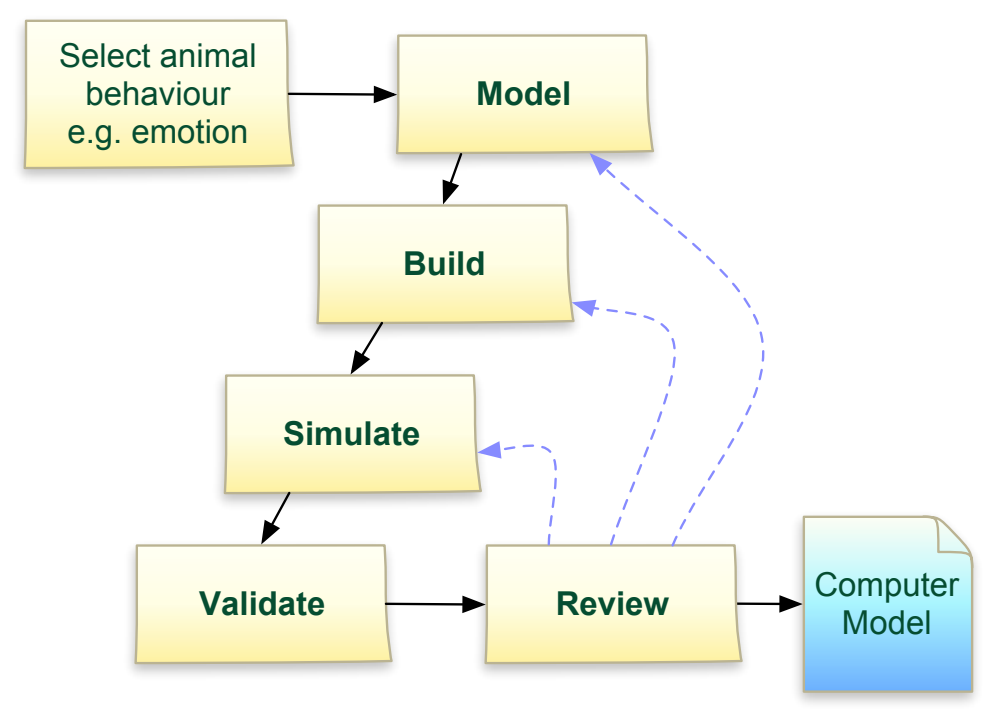

Figure 1: A common activity flow when developing cognitive models using computer programs.

\subsection{Motivation for emotion in technical systems}

- Objective: Produce technology for improving mission-level resilience in technical systems in broad domains (ASys - robust autonomy).

- This is an on-going work: 1) Looking for architectural inspiration in emotional mechanisms and 2) Not trying to build models to replicate biological behavior but to create universal architectural assets

Agents of advanced capabilities will not merely react to the present state of things but will make decisions concerning its many potential actions taking into account how the future states will be 2 . When the agent is at a particular time instant $t_{0}$ the selection of a concrete action alternative $A \in A_{a}, A_{b}, A_{c}$ will change the future; the agent shall anticipate the effects and determine the evolution of statedependent value along the system trajectory $x(t)$.

$$
V_{\text {expected }}=\int_{t_{0}}^{t_{f}} \operatorname{value}(x(t)) d t
$$




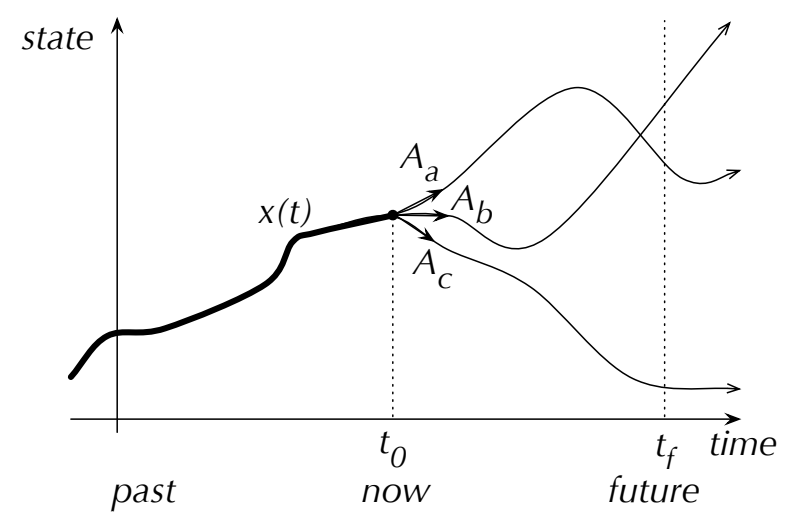

Figure 2: Sophisticated agents make decisions on what-to-do anticipating the future effects of its actions.

\section{Emotion in vivo and in silico}

\subsection{The many facets of emotion}

- Affective states, emotional states, cognitive impact, physiological impact, evolutionary development, emotional expression, emotional appraisal, etc.

- Are all those many aspects to be considered in machines?

\subsection{Four core aspects of emotion}

- Behavioral/expressive - Acting and externalisation of state

- Experiential/subjective - State valuation - qualia

- Somatic/neurophysiological — Bodily state

- Cognitive/interpretive - State representation - mental model

\subsection{Emotion Theories}

- Spreading activation models - Networks of nodes of cognitive-emotional bundles

- Componential models - Organisational variations in cognitive processes

- Parameter-based models - Appraisal dimensions that map to expressions

- From appraisal to external effects 


\subsection{Emotion beyond biology}

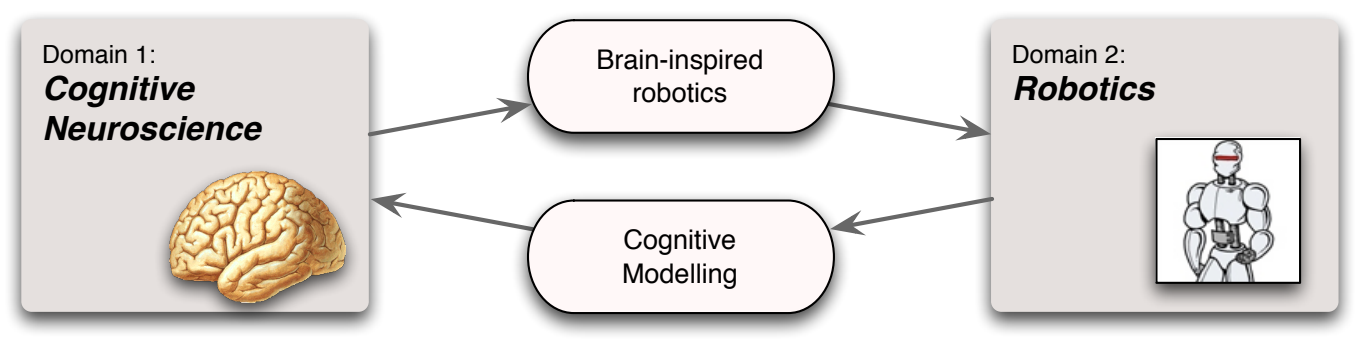

Figure 3: The path from biology to robotics.

\subsection{Emotion beyond biology}

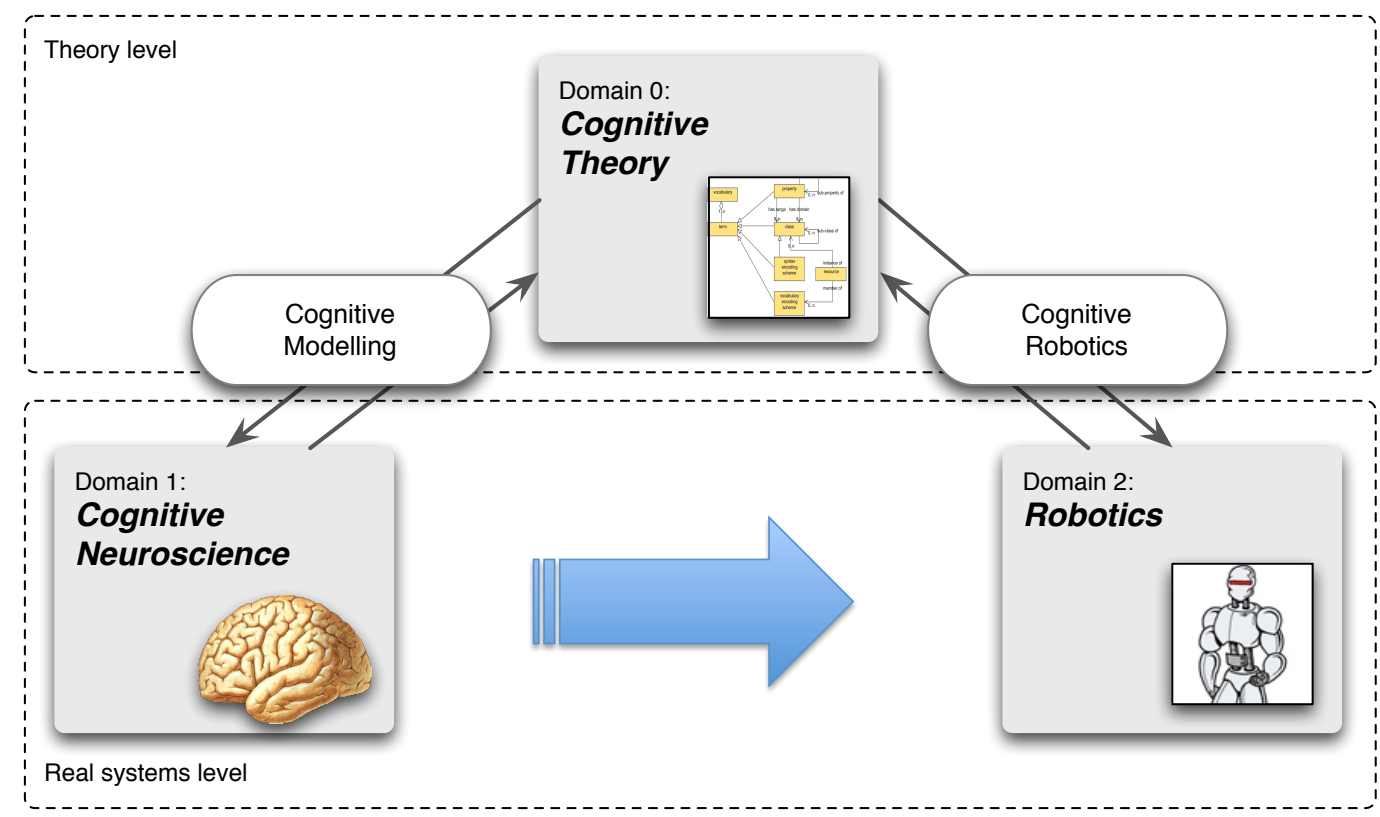

Figure 4: The path from biology to robotics shall go through general theory.

\subsection{A Stepped Program}

- Find fundamental architectural organisations

- Eliminate unnecessary details in the modeling of biological emotion 
- Consolidate in domain independent models

- Transfer to domains of technological use

\section{Adaptive systems}

\subsection{Emotion-Cognition-Autonomy}

- In the pursue of autonomy we investigate emotion-cognition integration architecture.

- Emotions are control architecture mechanisms directly serving the goals of the autonomous agent.

- They do so by providing real-time adaptation mechanisms to the agent.

\subsection{The issue of adaptation}

- Autonomous systems both natural and artificial do adapt:

- Animals - as species and individuals

- Machines - as objects and product lines

- Adaptation shall be framed in the context of autonomy:

$$
\text { Autonomy(agent, environment, task) }
$$

\section{Reconfiguration in Cognitive Systems}

The backpropagation algorithm ? offers a strategy for reconfiguration in architectures using the neural network paradigm.

Selfridge's Pandemonium offers a strategy for tuning the pandemonium based on signals flowing back from the cognitive demons layer into the computational demons layer.

Andrew uses the term "significance feedback" to refer to these processes ?.

It is not clear, however, if we are talking about learning, adaptation, reconfiguration, tuning, etc. when talking about non-static control structures ${ }^{1}$. The time

\footnotetext{
${ }^{1}$ The simple flow of perception is also a dynamical change. However, we may assume the persistence of some structure in this process in the very sense of Klir hypothetical structure.
} 
issue sees critical in all these phenomena (apart of what parts of the system are changing).

Here we hypotesize that the main difference between adaptation and emotion is the fact that the reconfiguration provided by emotion operates in real time?.

\subsection{Variable Structure Controllers}

Sliding mode control Edwards and Spurgeon (1998) — SMC — or variable structure control Zinober (1994) - VSC — are forms of nonlinear control developed as evolution of linear controllers to address their operational range limitations. These methods core nonlinear dynamics is produced by application of a switching control signal that forces a behavior that crosses a set of more "normal" dynamics. The control law, usually a state-feedback controller, is not a continuous function of time but a patchy one - continuously moving from one smooth patch to another.

In essence, the abstract structure of the controller -i.e. its control law- depends on the region of the state space where the system is. The controller switches control laws when crossing the borders of these regions. A common implementation is based on the fast switching among linear controllers at possibly very fast speeds. However the elementary controllers need not be linear and only continuity is required.

These controllers apply a strong control action -with practically infinite gain to force the behavior of the dynamic system to trajectories sliding along the restricted mode subspace - and must be applied with more care than other forms of more smooth and moderate nonlinear control. Due to design and implementation errors, the aggressive variable structure control action can lead to chatter, noise, energy losses, excitation of unmodelled dynamics and faults.

\subsection{Change and Timescale}

- Different timescales in dynamic change in agents: React/Control/Learn/Adapt

- Two levels of changes: State and Organisation

- Key: Structural adaptation

The dynamics of the agent-environment system is governed by the coupling of the dynamics of the world and the internal dynamics of the agent. The internal dynamics of the agent is determined by its organisation - $-a$ bundle of parts and their relations. 


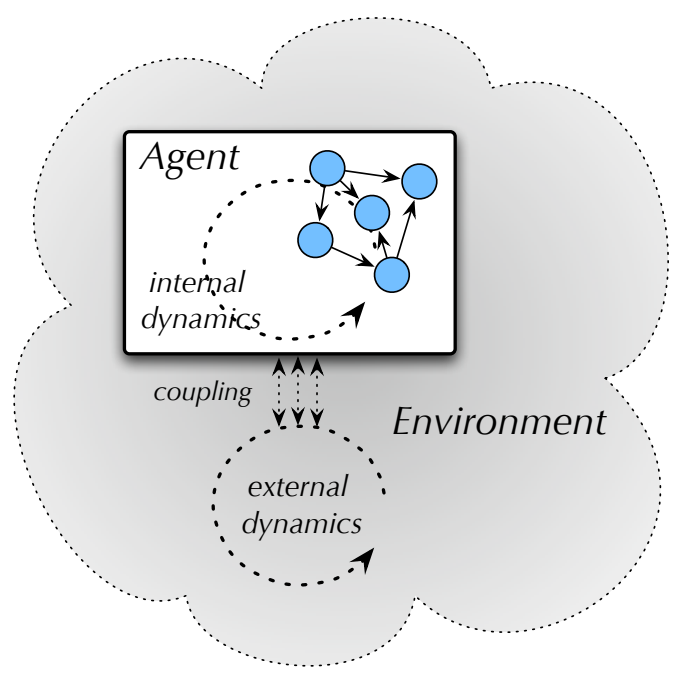

Figure 5: The dynamics of the agent-environment system is governed by the coupling of the dynamics of the world and the internal dynamics of the agent. 


\subsection{Structural Adaptation}

- By structural adaptation the agent changes its systemic structure: Agent $=$ Parts,Relations

- The changes may affect both the body and the mind

- Mind structural changes are easier and more flexible due to the plastic, informational nature of minds

\subsection{Fight or Flight}

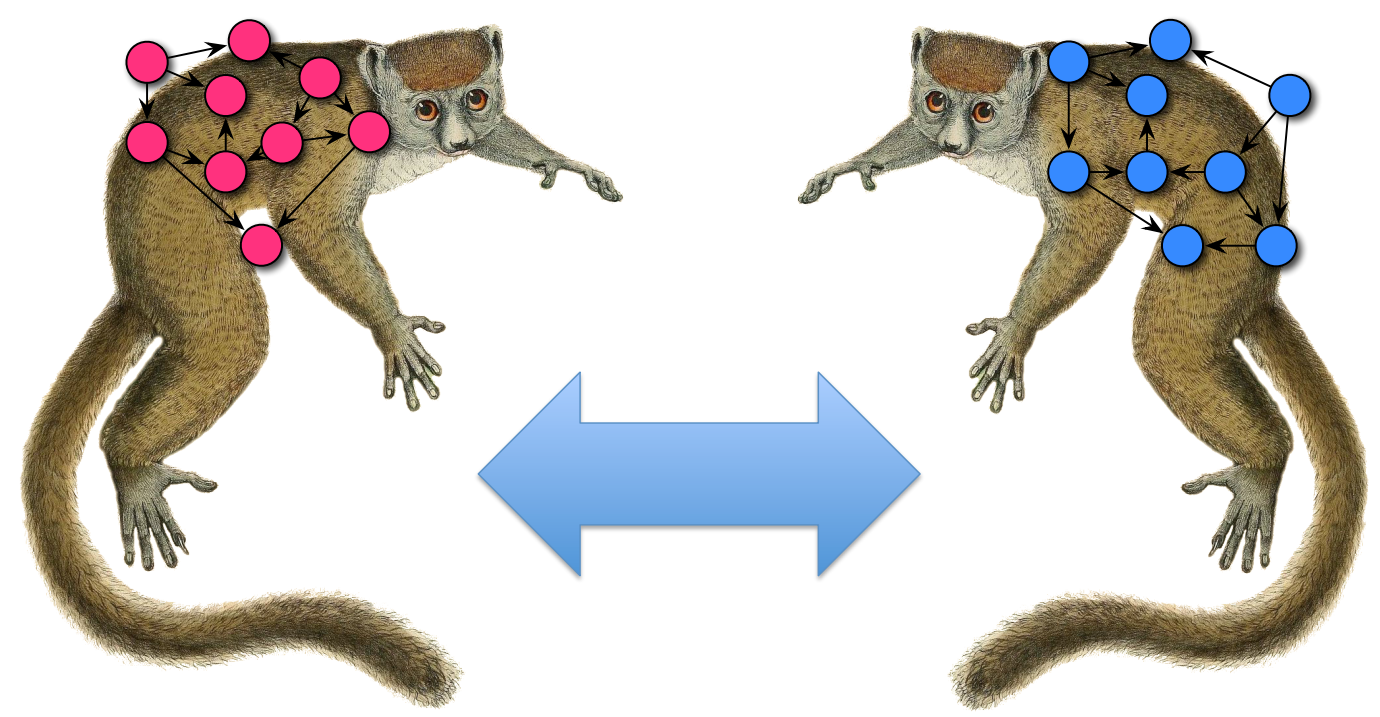

Figure 6: The concrete instantiation is selected dynamically.

\subsection{Architectural Change}

\subsection{Architectural Patterns}

- Design patterns - Functional organisations and Reusable strategies

- Architectural design patterns

- We look at emotion as an architectural pattern operating over over other architectural patterns ? a pattern-based pattern 

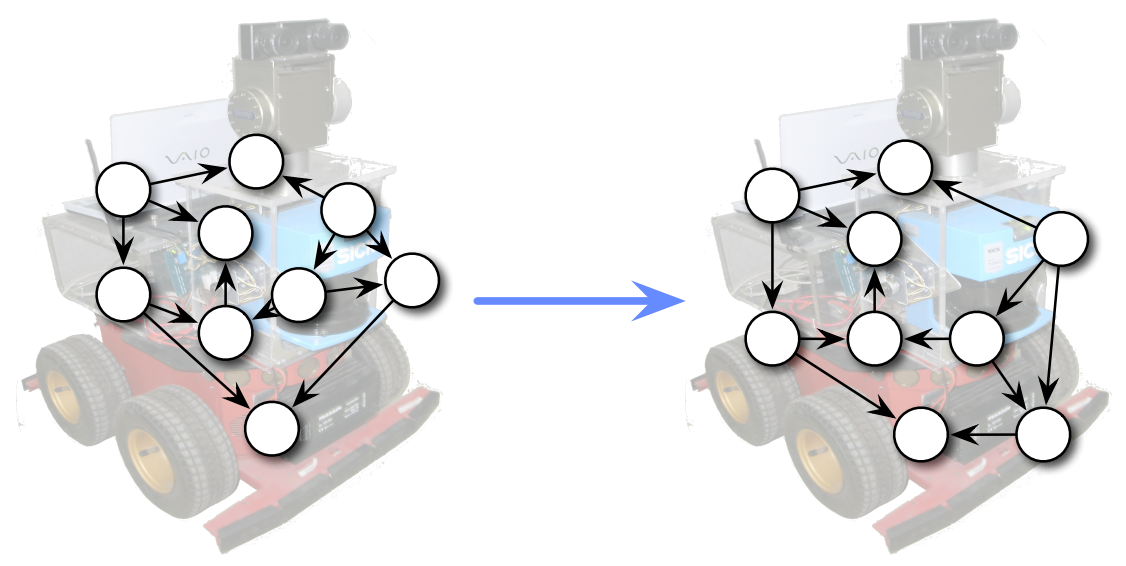

Figure 7: Architectural change offers the possibility of creating agents better tuned to environments.

\subsection{Taming the adaptation problem}

- There is a big difficulty of a general adaptation strategy based on structural change: the dimension of the space of organisational possibilities is too big (an inverse problem)

- It is possible to simplify the problem by means of the generation of operational patterns: forms of agent components? organisations that are tuned to specific niches/tasks

\subsection{Two basic processes}

- Functionalisation

- Aggregation and modularisation of subsystems (e.g. organs): Interface minimisation

- Homogenisation: use common integration infrastructure

- Patterning

- At the realisation - anatomical - level

- At the organisation - physiological - level 


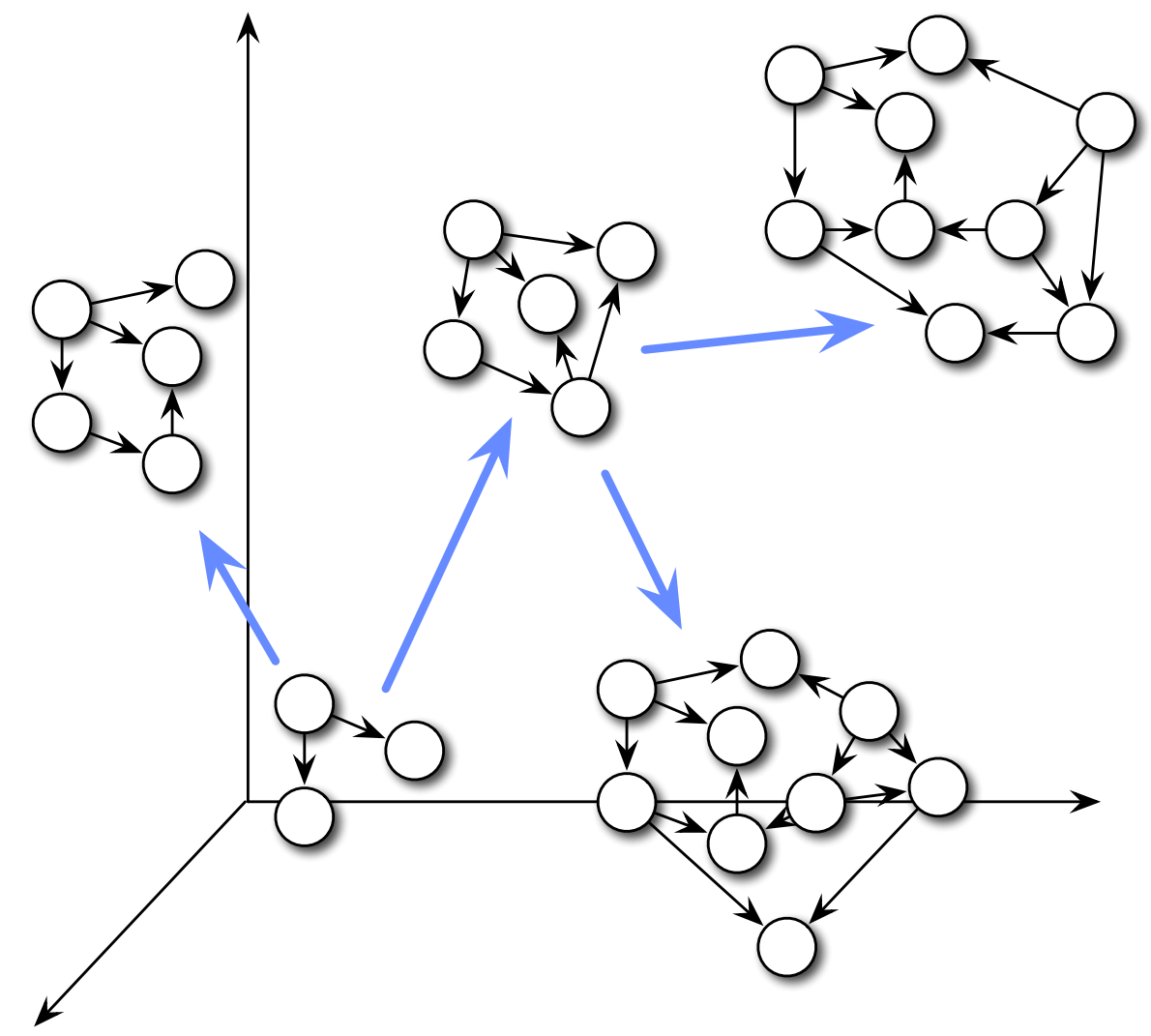

Figure 8: The space of architectural variation may be enormously complex. 


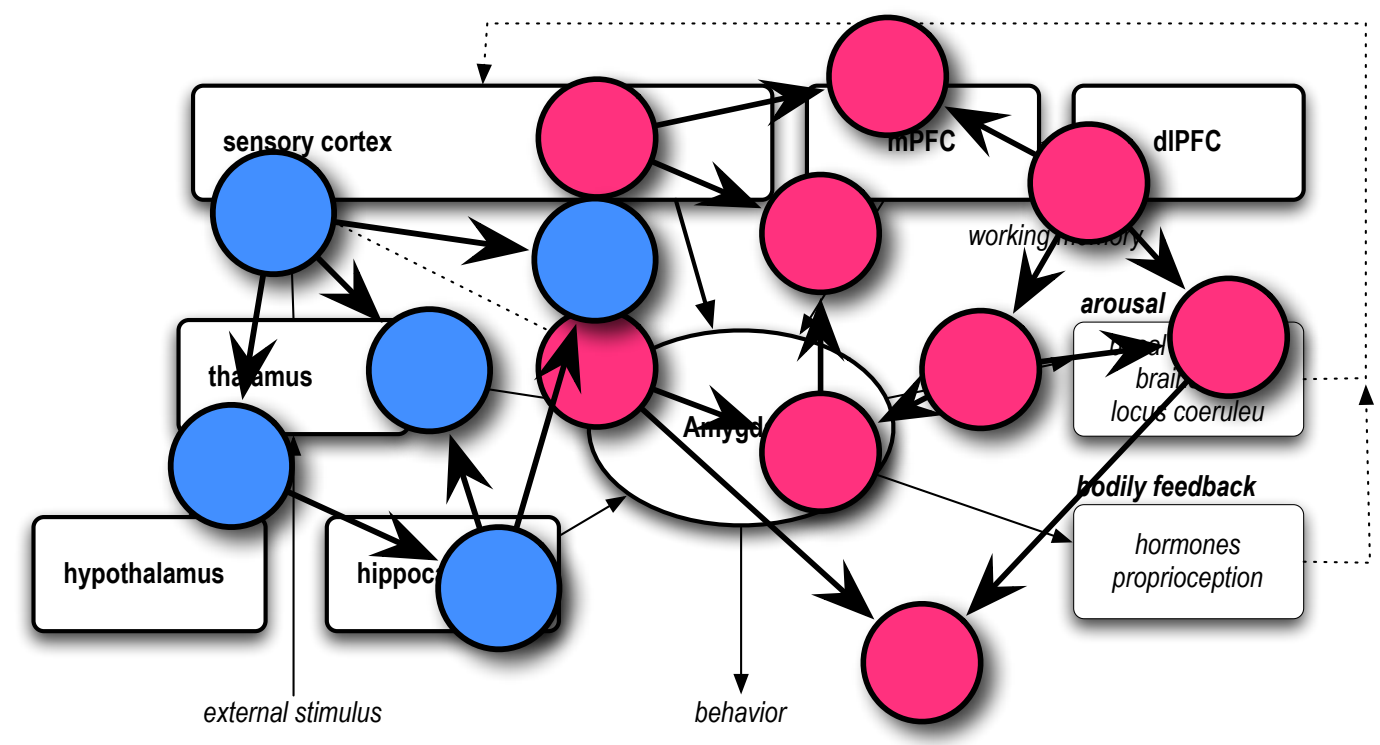

Figure 9: The physiological patterns are multiple-intanstiated over the anatomy of the agent.

\subsection{Anatomy/Physiology}

\section{Emotion as pattern-based metacontrol}

\subsection{What patterns to activate?}

- The decision concerning what control patterns shall be activated depends on an appraisal of the agent-environment situation in terms of agent goals.

- ?interrelated, synchronized changes in the states of all or most of the five organismic subsystems in response to the evaluation of an external or internal stimulus event as relevant to major concerns of the organism.?

\subsection{A View on Emotion}

- We understand emotion as a real-time adaptation mechanism based on the activation of predefined patterns of organization of the agent.

- These patterns have been produced (evolutionarily) for specific activities/niches

- In a sense, emotion selects the best available agent architecture for the present moment (esp. the control, informational part). 


\subsection{Emotion and Feedback}

- Emotion implements feedback from the evaluation of the degree of fitness between the functional state of the agent and the current state of affairs.

- This is broadcast as a synthetic global state

- These signals -in several appraisal dimensions- perform system-wide adaptation to achieve a multi-level communication of simplified but high impact information

\subsection{The Emotion Bus}

- Emotions are system-wide signals implementing this reconfiguration process ? an emotional bus.

- When represented at the level of awareness they constitute the emotional feelings.

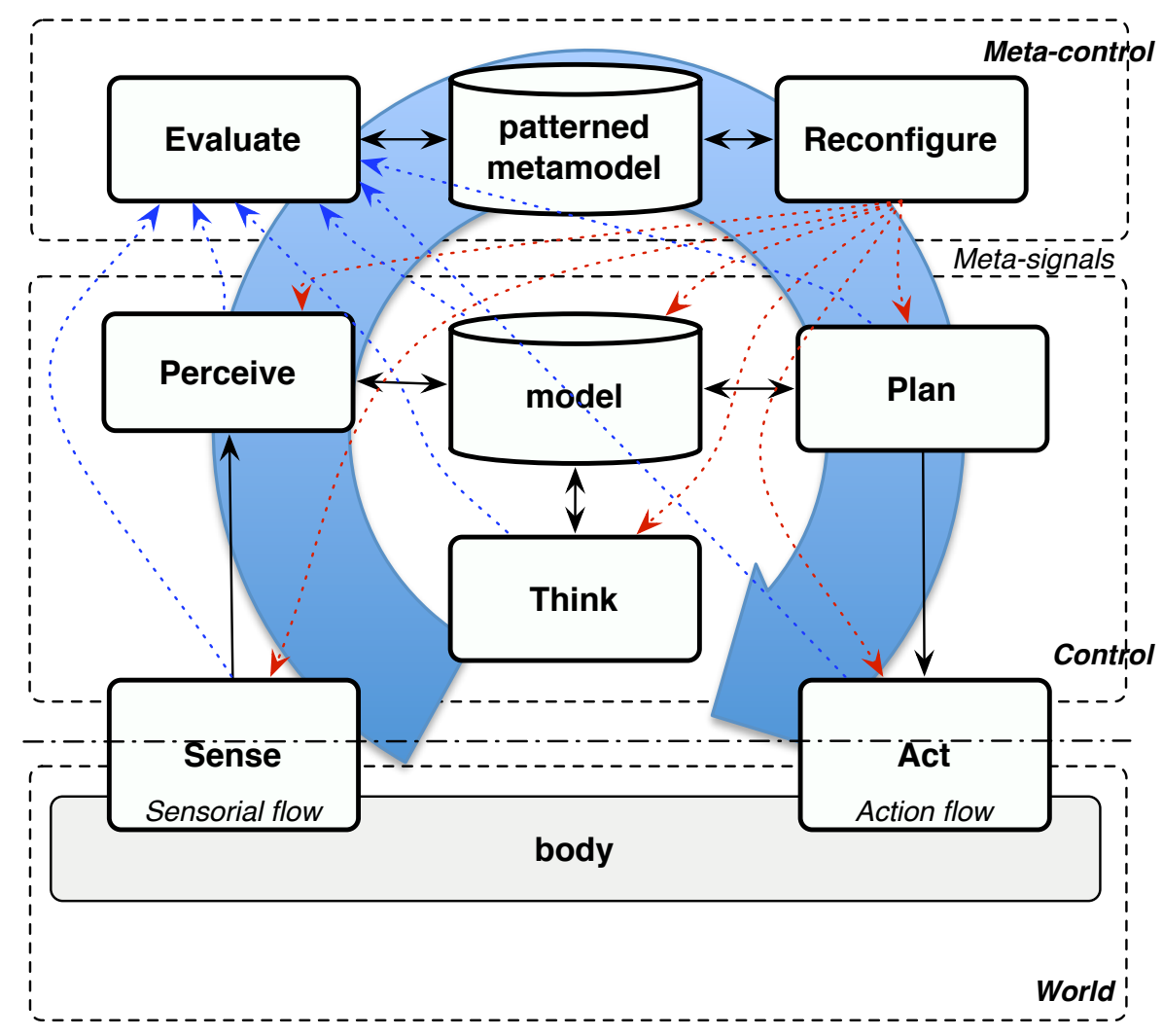

Figure 10: The metacontrol bus. 


\subsection{Two Cognitive Loops}

- The control level

- The metacontrol level

The control implements a target closure: controls domain magnitudes

The metasystem implements a functional closure: perceives and controls function

\subsection{Heterophenomenology}

- Emotion capture the pursue of goals by leveraging phenomenality.

- In building experiencing machines, engineering the right phenomenological mechanism is crucial because it will be the origin of the intrinsic motivations of the agents.

- We shall adopt an heterophenomenological engineering approach to build phenomenologies into machines to match our very own needs.

\section{Science and technology}

\subsection{Model Evaluation}

- This model can be evaluated in terms of both:

- Its potentiality as an architectural asset for building better machines

- Its explanatory power of the natural emotion

- For the first it must offer a path into technological consolidation as designs and reusable assets.

- For the second it must address specific traits of human emotion: appraisal, cognitive integration, emotion regulation, conscious and unconscious emotions, etc. Lots of painstaking work needed.

\subsection{Mapping into Machines}

- Mapping the model into machine realisations imply the construction of modular block-based cognitive systems ? issues of granularity

- Shall be deployed over a plastic integration infrastructure 
- Modern software architectural platforms helps with this (esp. distributed, real-time system platforms)

\subsection{Cognitive Functional Metacontrol}

\subsection{Technological path}

- Leveraging a technology of artificial emotion

- Two major issues:

- The organisational patterns: Persistent patterns and dynamic patterns; Some may be reverse-engineered from biological/brain systems

- The plastic engineering infrastructure: Componential, Self-referential, Run-time changeable, Real-time, Multigranular

\subsection{Current state}

- Done: Phase 0 ad-hoc implementation of rule-based metacontrol over rigid control patterns. Modular controllers based on instrumented widely available robot software.

- Ongoing: Phase 1

- Explicit function representation and model-based reasoning

- Bus-based emotion architecture

- Rigorous ontology for cognitive-emotional systems

- Future: Pattern learning + Consciousness

\subsection{Problems and issues}

- Dual representation of function in both implementational and goal terms

- Emotion dynamics - pattern change

- Concurrent emotions and emotion composition - pattern coexistence, integration and interference

- Heterogeneous agent emotions - ToM, societies

- Perception of the emotional state - persistent questions of self and qualia 


\subsection{Conclusions}

- Emotions are seen as processes of goal-centric metacontrol of patterned control architectures of autonomous agents.

- These processes happen at many levels ?e.g. at the conscious and unconscious levels. The agent can perceive its very own emotional processes ? having emotional qualia.

- This model can be implemented and leveraged in technical systems ? esp. in complex control systems.

- Current robotic architectures and infrastructures are in general too simple to fully leverage this vision.

- Several theoretical issues still open.

Zhong and DeLoach (2006)

\section{References}

Anderson, J. R., Bothell, D., and Byrne, M. D. (2004). An integrated theory of the mind. Psychological Review, 111(4):1036-1060.

Edwards, C. and Spurgeon, S. (1998). Sliding Mode Control: Theory and Applications. Taylor and Francis, London.

Hudlicka, E. (2009). Challenges in developing computational models of emotion and consciousness. International Journal of Machine Consciousness, 1(1):131-153.

Sander, D., Grandjean, D., and Scherer, K. R. (2005). A systems approach to appraisal mechanisms in emotion. Neural Networks, 18(4):317 - 352. Emotion and Brain.

Sanz, R. and López, I. (2000). Minds, MIPS and structural feedback. In Performance Metrics for Intelligent Systems, PerMIS '2000, Gaithersburg, USA.

Sanz, R., Matía, F., and Galán, S. (2000). Fridges, elephants and the meaning of autonomy and intelligence. In IEEE International Symposium on Intelligent Control, ISIC'2000, Patras, Greece.

Scherer, K. R. (2009). Emotions are emergent processes: they require a dynamic computational architecture. Philosophical Transactions of the Royal Society B: Biological Sciences, 364(1535):3459-3474.

Sun, R., editor (2008). The Cambridge Handbook of Computational Psychology. Cambridge University Press, New York. 
Zhong, C. and DeLoach, S. A. (2006). An investigation of reorganization algorithms. In Arabnia, H. R., editor, Proceedings of the 2006 International Conference on Artificial Intelligence, ICAI 2006, pages 514-517, Las Vegas, Nevada, USA. CSREA Press.

Zinober, A. S., editor (1994). Variable Structure and Lyapunov Control. SpringerVerlag, London.

Fellous,J.M. (2004) From Human Emotions to Robot Emotions, AAAI Spring Symposium on Architectures for Modeling Emotion, Stanford University, CA.

K. Fua, I. Horswill, A. Ortony, and W. Revelle (2009), "Reinforcement Sensitivity Theory and Cognitive Architectures," Proceedings of the AAAI Fall Symposium on Biologically Inspired Cognitive Architectures (AAAI Technical Report FS-09-01), pp. $52-54$

Hudlicka "Guidelines for Developing Computational Models of Emotions". International Journal of Synthetic Emotions, 2(1), 2011.

E. Hudlicka. Beyond cognition: modeling emotion in cognitive architectures. In sixth International Conference in Cognitive Modeling, ICCM-04, pages 118-123, 2004.

Utkin, V.I. (1992). Sliding Modes in Control and Optimization. Springer-Verlag. ISBN 978-0387535166. 